九州大学学術情報リポジトリ

Kyushu University Institutional Repository

\title{
The role of cathepsin $E$ in terminal differentiation of keratinocytes
}

\section{Kawakubo, Tomoyo}

Proteolysis Research Laboratory, Graduate School of Pharmaceutical Sciences, Kyushu University

Yasukochi, Atsushi

Proteolysis Research Laboratory, Graduate School of Pharmaceutical Sciences, Kyushu University

Okamoto, Kuniaki

Department of Dental Pharmacology, Graduate School of Biomedical Sciences, Nagasaki University

Okamoto, Yoshiko

Department of Biochemistry, Daiichi University College of Pharmaceutical Sciences

他

http://hdl. handle. net/2324/25561

出版情報: Biological Chemistry. 392 (6), pp.571-585, 2011-06. Walter de Gruyter GmbH バージョン:

権利関係: (C) 2011 Walter de Gruyter GmbH 
The role of cathepsin $\mathrm{E}$ in terminal differentiation of keratinocytes

\author{
Tomoyo Kawakubo ${ }^{1, a}$, Atsushi Yasukochi ${ }^{1,2, a}$, Kuniaki Okamoto ${ }^{3}$, Yoshiko \\ Okamoto $^{4}$, Seiji Nakamura ${ }^{2}$, and Kenji Yamamoto ${ }^{1, *}$
}

${ }^{1}$ Proteolysis Research Laboratory, Graduate School of Pharmaceutical Sciences, Kyushu University, Higashi-ku, Fukuoka 812-8582, Japan, ${ }^{2}$ Department of Oral and Maxillofacial Surgery, Graduate School of Dental Science, Kyushu University, Fukuoka, Japan, ${ }^{3}$ Department of Dental Pharmacology, Graduated School of Biomedical Sciences, Nagasaki University, Nagasaki 852-8588, Japan, ${ }^{4}$ Department of Biochemistry, Daiichi University College of Pharmaceutical Sciences, Fukuoka 815-8511, Japan.

${ }^{\mathrm{a}}$ These authors contributed equally to this work.

*Corresponding author: Kenji Yamamoto, Proteolysis Research Laboratory, Graduate School of Pharmaceutical Sciences, Kyushu University, Higashi-ku, Fukuoka 812-8582, Japan. Phone/Fax: +81-92-642-6768, E-mail: kyama@dent.kyushu-u.ac.jp

Running title: Cathepsin E in Keratinocyte Differentiation 


\begin{abstract}
Cathepsin E (CatE) is predominantly expressed in the rapidly regenerating gastric mucosal cells and epidermal keratinocytes, besides the immune system cells. However, the role of CatE in these cells remains unclear. Here we report a crucial role of CatE in keratinocyte terminal differentiation. CatE deficiency in mice induced abnormal keratinocyte differentiation in the epidermis and hair follicle characterized by the significant expansion of corium and the reduction of subcutaneous tissue and hair follicle. In a model of skin papillomas formed in three different genotypes of syngeneic mice, CatE deficiency resulted in the significantly reduced expression and altered localization of the keratinocyte differentiation-induced proteins keratin 1 and loricrin. The link of CatE to the regulation of the expression of epidermal differentiation-specific proteins was corroborated by in vitro studies with primary cultures of keratinocytes from the three different genotypes of mice. In the wild-type keratinocytes after differentiation-inducing stimuli, the expression profile of CatE gene was compatible with those of the terminal differentiation marker genes tested. Over-expression of CatE in mice enhanced the rate of keratinocyte terminal differentiation process, whereas CatE deficiency resulted in the delayed differentiation accompanying the reduced expression or the ectopic localization of the differentiation markers. Our findings suggest that CatE in keratinocytes functionally links to the expression of the terminal differentiation markers, thereby regulating the formation and homeostasis of the epidermis.
\end{abstract}

Key words: Aspartic proteinase; Cathepsin E; Keratinocyte; Skin Papilloma; Terminal differentiation 


\section{Introduction}

The epidermis is a stratified and keratinized squamous epithelium mainly composed of keratinocytes. Epidermal differentiation results in formation of several distinct cell layers characterized by their ultrastructure, mitotic state and expression of specific markers. The basal keratinocytes have a high proliferating potential and are characterized by specific expression of keratin 5 (K5) and K14, as well as by cuboidal structure and attachment to the underlying basement membrane. It has long been considered that the basal keratinocytes migrate to the suprabasal layer and directly become spinous cells expressing K1, K10 and involucrin when terminal differentiation is initiated. However, recent evidence has shown that most of basal keratinocytes do not really migrate, but arise from dividing basal layer cells in which the mitotic spindle is perpendicular to the basement membrane, so resulting in the development of suprabasal cells that commit to enter a program of terminal differentiation (reviewed in Blanpain and Fuchs, 2009). The spinous cells concomitantly switch from the expression of such differentiation markers as $\mathrm{K} 1, \mathrm{~K} 10$ and involcrin to the expression of the late differentiation markers loricrin and filaggrin according to the levels of differentiation, thereby forming the granular layer characterized by the existence of keratohyalin granules. The granular keratinocytes terminally differentiate to form the cornified layer 
at outmost layer, which consists of extremely flat, keratin-filled and anucleated keratinocytes and is believed to be primary responsible for the barrier function of the skin. Dermatological disorders ranging from minor cosmetic problems to life-threatening conditions are commonly due to abnormal differentiation of the keratinocytes. Elucidation of the intracellular molecules involved in the cellular differentiation processes and the regulation of epidermal homeostasis is, thus, of special importance for the understanding and therapy of these disorders.

Recent analyses of patients with defective protease genes (Toomes et al., 1999, Chavanas et al., 2000) and mouse mutants with defects in proteases (Roth et al., 2000; List et al, 2002; Egberts et al., 2004, Demerjian et al., 2008) have highlighted their crucial roles in the regulation of dermal differentiation and the maintenance of epidermal homeostasis (reviewed in Ovaere et al., 2009). Several studies have also indicated the importance of protease inhibitors in dermal differentiation using mouse mutants with their defects (Sundberg et al., 1997; Zeeuwen et al., 2002). Accordingly, it is likely that the protease-inhibitor balance is an important factor for normal progression of dermal differentiation processes (reviewed in Zeeuwen 2004, Candi et al., 2005).

We have previously identified two analogous aspartic proteinases of the pepsin superfamily, CatE and cathepsin D (CatD), in human and rat epidermis (Igarashi et al., 
2004; Sakai et al., 1989; Hara et al., 1993). Both enzymes have similar enzymatic properties, but their tissue distribution and cellular localization were apparently different (reviewed in Yamamoto, 1999). In rat and human skin, CatD was concentrated in granular cells, whereas CatE was diffusely detected in the cytoplasm of almost all epidermal cells (Hara et al., 1993). Additionally, immunoelectron microscopic studies demonstrated that CatD was localized mainly on desmosomes of human stratum corneum, whereas CatE was present intracellularly within the squames (Igarashi et al., 2004). These observations strongly suggest that these enzymes have different functions in dermal differentiation processes. Recently, Egberts et al. (2004) demonstrated that CatD deficiency in mice resulted in the reduced transglutaminase 1 activity essential for the correct cornified-envelope formation and the reduced expression of the cornified envelop proteins involucrin and loricrin, thereby suggesting a functional link of CatD to the regulation of epidermal differentiation. On the other hand, recent studies have demonstrated that $\mathrm{CatE}^{-/-}$mice spontaneously develope atopic dermatitis-like skin lesions when reared under conventional conditions, but not under specific pathogen-free conditions (Tsukuba et al., 2003), and increase in susceptibility to hapten-induced experimental dermatitis (Tsukuba et al., 2003) and to bacterial infection (Tsukuba et al., 2007), suggesting that CatE plays a crucial role in the protection of against various 
environmental stresses, such as water loss and microorganism infection. These observations also suggest that CatD and CatE are differentially involved in keratinocyte differentiation and maintenance of dermal homeostasis. Hence to assess the role of CatE in multistage processes important for dermal differentiation, we manipulate this protein in both in vivo and in vitro experiments using a model of skin papilloma induced in three different genotypes of syngeneic mice, CatE-deficient $\left(\mathrm{CatE}^{-/}\right)$, wild-type $\left(\mathrm{CatE}^{+/+}\right)$, and CatE-overexpressing transgenic $\left(\mathrm{CatE}^{\mathrm{Tg}}\right)$ and primary cultures of keratinocytes from each genotype of mice, respectively.

\section{Results}

\section{CatE deficiency induces abnormal differentiation processes in epidermis and hair}

\section{follicle in vivo}

We used three different genotypes of the syngeneic C57BL/6 mice at three weeks of age, $\operatorname{CatE}^{-/-}(\mathrm{n}=8), \operatorname{CatE}^{+/+}(\mathrm{n}=5)$, and $\operatorname{CatE}^{T g}(\mathrm{n}=5)$. No significant differences in gross appearance and body size were detected between $\mathrm{CatE}^{+/+}$and $\mathrm{CatE}^{\mathrm{Tg}}$, but $\mathrm{CatE}^{-/-}$mice were apparently smaller than other genotypes (data not shown). Also, the epidermis and hair follicles of $\mathrm{CatE}^{-/-}$mice showed abnormalities in epidermal differentiation processes, which were characterized by the more expanded corium and the significantly 
reduced subcutaneous tissue and hair follicle compared with those of wild-type littermates (Fig. 1A). Given that hair follicles undergo repeated cycles of proliferation and differentiation (anagen stage), apoptosis (catagen stage) and resting (telogen stage) of epithelial cells (Stenn and Paus, 2001), we performed quantitative evaluation of hair follicle stages on the basis of well-defined morphological criteria (Muller-Rover et al, 2001). As shown in Fig. 1A, the majority of hair follicles in each genotype of mice at 3 weeks of age were in the telogen stage. We also found that a significant number of hair follicles in $\mathrm{CatE}^{+/+}$mice at this age were in the transitional process making preparations for anagen stage, whereas $\mathrm{CatE}^{-/}$mice had little or no hair follicle in the anagen stage at the same age. By contrast, the percentage of hair follicles in the catagen stage was very low in $\mathrm{CatE}^{+/+}$mice, whereas that was high in $\mathrm{CatE}^{-/}$mice. Notably, little or no significant infiltration of effector cells such as neutrophils, macrophages and lymphocytes into the epidermis of $\mathrm{CatE}^{-/-}$mice was noted (Fig. 1A). Meanwhile, the extent of infiltration of these cells into the dermis of $\mathrm{CatE}^{-/}$mice was not significantly different from that of $\mathrm{CatE}^{+/+}$mice. These findings indicate that the abnormalities in $\mathrm{CatE}^{-/}$epidermis were not secondary inflammatory responses due to CatE deficiency and strongly suggest that CatE may be involved in the formation of epidermal barrier and the development of hair follicles. These results thus prompted us to investigate 
specific functions of CatE in epidermal differentiation. To explore this issue, we first determined whether and which cell types in mouse skin express CatE. For this purpose, we prepared the primary culture of skin keratinocytes from $\mathrm{CatE}^{+/+}$mice and analyzed the cell extract by Western blotting under reducing conditions. CatE was expressed mainly as a $46-\mathrm{kDa}$ proenzyme and slightly as a $42-\mathrm{kDa}$ mature form (Fig. 1B). On the other hand, CatE expression in hair follicles was determined by in situ hybridization (ISH) and was found to be expressed and localized in close vicinity to the inner root sheath (Fig. 1D), which was consistent with the previous observation that CatE was expressed in the cuticle of the keratogenous zone (Ishimatsu-Tsuji et al., 2005).

Characterization of skin papillomas generated in each genotype of mice by DMBA/TPA treatment

The first tumors generated by two-stage mouse skin tumorigenesis protocol using the initiater 7, 12-dimethylbenzanthracene (DMBA) and the promoter 12-Otetradecanoylphobol-13-acetate (TPA) have been demonstrated to be premalignant lesions, called papillomas (reviewed in Klein-Szanto, 1989), which further progress to squamous cell carcinoma at different rates by the continuous TPA application (Aldaz et al., 1987; Burns et al., 1976). Histopathological analysis of surgical specimens of tumor lesions of each genotype revealed no distinct invasion or intravascular metastasis in 
tumors generated in each genotype of mice (Fig. 2B, E, H), indicating that most of the tumors were premalignant under the conditions employed. All of the $\mathrm{CatE}^{+/+}$and $\mathrm{CatE}^{\mathrm{Tg}}$ mice induced multiple skin papillomas, whereas more than $20 \%$ of $\mathrm{CatE}^{-/-}$mice did not generate the papillomas under the conditions used (Fig. 3A, B). The number of papillomas per mouse was positively associated with the expression levels of CatE, increasing according to the rank order $\operatorname{CatE}^{-/-}<\mathrm{CatE}^{+/+}<\mathrm{CatE}^{T g}$ mice, with the effect of CatE expression on the incidence of papillomas per $\mathrm{CatE}^{T g}$ mice being about 12 times that apparent for $\mathrm{CatE}^{-/-}$mice (Fig. 3C). The size of papillomas in $\mathrm{CatE}^{+/+}$mice was smaller than that in $\mathrm{CatE}^{T g}$ mice (Fig. 3D). $\mathrm{CatE}^{-/}$mice developed little papilommas, but their size was equal to or more than that in $\mathrm{CatE}^{+/+}$mice, suggesting that papilloma size was negatively associated with the expression of CatE, decreasing according to the rank order $\mathrm{CatE}^{-/-}>\mathrm{CatE}^{+/+}>\mathrm{CatE}^{\mathrm{Tg}}$ mice.

Histological analysis also revealed that the extent of keratinocyte differentiation, called keratinization, was positively associated with the expression levels of CatE, increasing according to the rank order $\operatorname{CatE}^{-/-}<\mathrm{CatE}^{+/+}<\mathrm{CatE}^{T g}$ mice (Fig. 2A, D, G). The papillomas of $\mathrm{CatE}^{-/-}$and $\mathrm{CatE}^{+/+}$mice contained a significant number of cells indicating mitotic divisions, which may be progressing to squamous cell carcinoma (Fig. 2C, F, I). By contrast, the cells showing mitotic divisions were scarcely found in the 
papillomas of $\mathrm{Cat}^{T g}$ mice. It is also noted that papillomas of CatE ${ }^{T g}$ mice but not $\mathrm{CatE}^{-/}$and $\mathrm{CatE}^{+/+}$mice contained a large number of tumor-infiltrating effector cells such as neutrophils (Fig. 2I). These results strongly suggest that a significant decrease in the number of papillomas in $\mathrm{CatE}^{-/-}$mice is due to altered epidermal differentiation processes, whereas the appearance of multiple papillomas in $\mathrm{CatE}^{T g}$ mice is likely due to stimulation of the keratinocyte differentiation rate by the increased expression of CatE. Taken together, it is assumed that Cat $\mathrm{E}$ is associated with differentiation-leading keratinization and enhanced infiltration of inflammatory cells into tumorous parenchyma to remove malignant squamous cells, probably developed from occasional papillomas.

\section{Expression of differentiation-specific markers in normal skin and papillomas}

Epidermal differentiation is known to begin with proliferation of basal keratinocytes and to end with the formation of the cornified layer. The process of keratinocyte proliferation and differentiation is also characterized by the expression of specific marker proteins. To determine whether epidermal differentiation was altered by the CatE expression level, we thus analyzed the protein expression and localization of differentiation-specific proteins in the epidermis of each genotype of mice by immunohistochemical studies. K1 is one of the proteins to be expressed during 
keratinocyte differentiation. Control $\mathrm{CatE}^{+/+}$mice treated with acetone without DMBA and TPA did not result in papilloma formation (Fig. 4 and Table 1). K1 staining in skin of the control mice was exclusively confined to the outer cell layer, hair follicles and sebaceous glands (Fig. 4A), which was comparable with that reported previously (Kartasova et al., 1993). Both horny layer and epidermis of $\mathrm{CatE}^{+/+}$mice treated with DMBA and TPA showed the similar K1 staining patterns to those obtained with the control mice. K1 staining was also observed in all the papillimas of each genotype of mice (Fig. 4A). No significant differences in the extent of K1 expression in normal skin regions and papillomas of both the horny layer and the epidermis were detected among genotypes. Notably, $\mathrm{CatE}^{-/-}$mice treated with DMBA and TPA displayed the increased ectopic expression and localization of $\mathrm{K} 1$ in the muscle layer, implying abnormal keratinocyte differentiation by CatE deficiency. Loricrin is a main component of the epidermal cornified envelop proteins and localized in the granular layer during cornification of human epidermis (Sundberg, JP., 1994; Tiano, HF., 2002). Loricrin staining was found in the terminal differentiated horny layer of $\mathrm{CatE}^{+/+}$skin treated with acetone alone (Fig. 4B). The expression and localization of loricrin was significantly reduced in normal skin regions in the horny layer of $\mathrm{CatE}^{+/+}$mice treated with DMBA and TPA (Table 1) and inversely increased in papillomas formed in these mice (Fig. 4B 
and Table 1). CatE deficiency induced the significantly decreased expression of loricrin in both normal and papillomous regions in the horny layer by DMBA/TPA treatment and the markedly increased ectopic localization in the muscle layer, also suggesting abnormal keratinocyte differentiation by CatE deficiency. These data thus indicate a close link of CatE expression to keratinocyte differentiation. K13 expression is known to not occur simultaneously with keratinocyte differentiation but follow squamous cell differentiation (Sutter, C., 1991). Thus K13 is likely to be expressed and localized mainly in differentiating and/or differentiated keratinocytes of non-cornifying squamous epithelia. As shown in Figure 4C, K13 expression was found in cells mainly in the granular layer and epidermis of $\mathrm{CatE}^{+/+}$and $\mathrm{CatE}^{T g}$ papillomas, and partly in the hair follicle of the control mice treated with acetone alone. Notably, the extent of K13 expression in papillomas in the horny layer and epidermis of $\mathrm{CatE}^{-/-}$mice was very low compared with that of other genotypes. The expression and localization of K13 in the papillomas generated in the horny layer of DMBA/TPA-treated $\mathrm{CatE}^{+/+}$and $\mathrm{Cat}^{\mathrm{Tg}}$ mice was significantly reduced by CatE deficiency. Given that K13 is an early marker of DMBA/TPA-induced papilloma progression (Robles et al., 1993, Gimenez-Conti et al., 1990), CatE appeared to be essential for the papilloma promotion step in the process of keratinocyte terminal differentiation. The in vivo experiments thus suggest that a 
significant decrease in the number of papillomas in $\mathrm{CatE}^{-/-}$mice is due to the altered expression of the epidermal differentiation proteins, thereby leading to the impaired keartinocyte terminal differentiation.

\section{Correlation of CatE expression and keratinocyte terminal differentiation in primary cultures of keratinocytes}

To determine whether or not the pathohistological effects observed in skin papillomas of $\mathrm{CatE}^{-/-}$mice using DMBA and TPA was actually related to altered expression of CatE, we prepared primary cultures of epidermal keratinocytes from the three different genotypes of newborn syngeneic mice. We first examined the survival of these cells in response to DMBA treatment, because this carcinogen is known to induce genetic defects and aberrant signaling pathways and ultimately apoptosis in the damaged cells (Kim et al., 2009). When treated with acetone alone, primary keratinocyte cultures of each genotype had only a small amount of annexin V-and/or propidium iodide-positive cells and showed no significant differences in the extent of cell viability (Fig. 5). In addition, DMBA treatment had little effect on the viability of keratinocytes from each genotype at any of the doses used. These data indicate that the DMBA-induced initiation step in keratinocyte papillomagenesis is scarcely affected by CatE expression levels and that the increased number of papillomas in $C a t E^{T g}$-mice induced by 
DMBA/TPA treatment is unlikely due to either enhancement in DMBA-induced keratinocyte proliferation or reduction in the apoptosis. We next examined the correlation between CatE expression and keratinocyte differentiation in TPA-treated cultured keratinocytes from each genotype. TPA is known to act as an efficient agent inducing homogenous proliferation and differentiation of mouse keratinocytes that are not treated with DMBA (Leder et al., 1990; Dlugosz et al., 1993). Quantitative RT-PCR analysis using TPA-treated primary cultured keratinocytes revealed that, while the expression levels of $K 1$ and loricrin was relatively low in untreated CatE $E^{-/-}$keratinocytes, the mRNA expression of both genes was strongly stimulated in $\mathrm{CatE}^{+/+}$keratinocytes 48 and $72 \mathrm{~h}$ after treatment with TPA (Table 2). A strong stimulation of the expression of K1 and loricrin was also detected in cultured $\mathrm{CatE}^{\mathrm{Tg}}$ keratinocytes 48 and $72 \mathrm{~h}$ after treatment with TPA. Notably, the mRNA expression of loricrin as a marker of terminal epidermal differentiation or keratinization at 48 and $72 \mathrm{~h}$ after treatment with TPA was significantly higher in $\mathrm{CatE}^{\mathrm{Tg}}$ keratinocytes than in $\mathrm{CatE}^{+/+}$keratinocytes, whereas the mRNA expression of $K 1$ as a marker of the spinous and glanular layers of the epidermis in $\mathrm{CatE}^{T g}$ keratinocytes was comparable to that in $\mathrm{CatE}^{+/+}$keratinocytes. It is worth emphasizing that the expression of both genes is strongly inhibited in TPA-treated $\mathrm{CatE}^{-/-}$keratinocytes, although their expression profiles in themselves are similar to 
those of other genotypes of mice. To further prove the correlation between CatE expression and keratinocyte differentiation, we determined the expression of $K 13$, a TPA-mediated epidermal differentiation marker expressed in differentiating and/or differentiated keratinocytes, in each genotype of the cells. K13 expression was significantly increased in TPA-treated $\mathrm{Cat}^{+/+}$keratinocytes in a time-dependent manner and reached a plateau value at $48 \mathrm{~h}$ after the treatment. A strong stimulation of K13 expression was induced in $\mathrm{CatE}^{\mathrm{Tg}}$ keratinocytes compared with $\mathrm{CatE}^{+/+}$ keratinocytes, with the effect of TPA on K13 expression in the former cells at 48 and 72 $\mathrm{h}$ after the treatment being about 18 times that apparent for the latter cells. Although the terminal expression level of $K 13$ mRNA in TPA-treated CatE $^{-/-}$keratinocytes was comparable to that in $\mathrm{CatE}^{+/+}$keratinocytes, the rate of terminal differentiation of the former cells was apparently delayed compared with that of the latter cells, indicating that the keratinocyte differentiation process is exacerbated in the absence of CatE. These abnormal expression patterns of keratinocyte differentiation markers, K1 and loricrin, were also found to be induced when the cells were treated with the classical differentiation-inducing reagent, $\mathrm{Ca}^{2+}$ (data not shown). In agreement with RT-PCR results, Western blot analysis data revealed that Cat E, particularly the mature enzyme, was time-dependently increased in $\mathrm{Cat}^{+/+}$keratinocytes treated with TPA (Fig. 6). We 
thus confirmed that the altered expression and localization of keratinocyte differentiation markers observed in a model of skin papilloma by DMBA/TPA treatment was actually related to altered expression of CatE in epidermis.

\section{Discussion}

It has long been considered that the functions of CatE are confined to terminal and non-specific degradation of proteins in endosomal and/or lysosomal compartments. Accumulating evidence, however, suggests that this enzyme has additional, more specific functions in vivo (Lees et al., 1990; Athauda et al., 1991; Kageyama, 1993; Kageyama et al., 1995; Henningsson et al., 2005; Shin et al., 2007). To identify specific in vivo functions of this enzyme, CatE-deficient mice were generated by gene-targeting and their phenotypic alterations were analyzed (Tsukuba et al., 2003; Tsukuba et al., 2006; Kakehashi et al., 2007; Kawakubo et al., 2007; Yanagawa et al., 2007). From the previous studies, it has been suggested that CatE contributes the maintenance of homeostasis through more specific functions in host defense mechanisms. CatE is known to be predominantly expressed in the rapidly regenerating gastric mucosal cells and epidermal keratinocytes, besides the immune system cells. However, specific functions of this enzyme in these cells remain speculative. In this study, we first 
demonstrate that CatE deficiency leads to some remarkable alterations in the epidermis and hair follicle, which were characterized by the moderately expanded corium, the reduced subcutaneous tissue, the reduced hair follicle and the dry skin. Pathological inflammatory responses were excluded as a putative cause of the observed skin and hair disorder. It has previously been shown that defects in the cornified cell envelop proteins such as transglutaminase 1 are found in human cornification disorders (Huber et al., 1995; Russel et al., 1995). In addition, various mouse mutants with defects in not only growth factors such as keratinocyte growth factor (Guo et al., 1996; Hebert et al., 1994) but also proteases such as cathepsins L (Roth et al., 2000) and D (Egberts et al., 2004) have been shown to display the spontaneous development of skin and hair disorders. However, the phenotypic alterations found by mutations with these genes were likely to be different from those by CatE deficiency. Thus the phenotypic alterations observed in the epidermis and hair follicle of $\mathrm{CatE}^{-/-}$mice prompted us to explore specific functions of CatE in epidermal differentiation processes.

In vivo experiments using a model of skin papillomas, we provide evidence that the CatE expression is correlated positively with the number of papillomas per mouse and negatively with their size. The present results that the sensitivity of DMBA to keratinocytes is comparable among the three different genotypes of mice suggest that 
CatE had little effect on the initiation step in papillomagenesis. Therefore, the remarkable resistance of $\mathrm{CatE}^{-/-}$mice to the papilloma development is rather related to altered TPA-mediated differentiation and proliferation processes. In an attempt to identify the role of CatE in epidermal differentiation, we first determined whether the expression of the differentiation-specific marker proteins was altered in papillomas of the respective genotypes of mice. The expression and localization of $\mathrm{K} 1$, a major protein to be expressed during an earlier cornification stage, in the horny layer and epidermis was independent of the expression levels of CatE, except that the ectopic expression and localization in the muscle layer were induced by CatE deficiency. The results strongly suggest that CatE participates in epidermal differentiation at a more advanced stage. On the other hand, the expression and localization of loricrin, a major cornified cell envelope protein of terminally differentiated epidermal cells, in papillomas in the horny layer and epidermis of $\mathrm{CatE}^{-/-}$mice were significantly reduced compared with those of other genotypes. Concurrently, a stimulation of the ectopic expression and localization in the muscle layer of $\mathrm{K} 1$ and loricrin was detected in $\mathrm{CatE}^{-/-}$mice. It is also noteworthy that the expression and localization of the terminal keratinocyte differentiation marker K13 were more intensely reduced in papillomas of $\mathrm{CatE}^{-/-}$mice compared with those of other genotypes. Given that the expression of K13 
is restricted to differentiating and differentiated keratinocytes, the reduced expression and localization of K13 in $\mathrm{CatE}^{-/-}$papillomas shows the functional link between CatE expression and epidermal terminal differentiation. In addition, our previous study demonstrated that the over-expression of CatE in mice resulted in a marked increase in the number and extent of tumor-infiltrating effector cells such macrophages within or close to tumors formed by injection of tumor cells, thereby leading to apoptosis in tumor cells (Kawakubo, T. et al., 2007). Therefore, an increased number of tumor-infiltrating effector cells in papillomas of $\mathrm{CatE}^{T g}$ mice may serve to eliminate transformed cells induced by DMBA/TPA treatment, and concurrently the enhanced CatE expression forced keratinocytes to develop benign papillomas. This may be one of the reasons for appearance of a small size of papillomas in $C a t E^{T g}$ mice. Papillomas generated in each genotype of mice under the conditions used were shown to be premalignant and there were little papillomas progressed to squamous carcinomas. However, the incidence of conversion of papillomas to squamous carcinomas was increased by prolonged application of TPA (data not shown). This was consistent with the previous report that a significant portion of papillomas generated by the initiation/promotion protocols progressed to squamous cell carcinoma 24 weeks after twice-weekly topical application of TPA (Dhawan et al., 1999; Fu et al., 2009). Together, 
the present in vivo results strongly suggest that CatE is essential for keratinocyte terminal differentiation required for the promotion step of papillomas.

The functional link of CatE to the expression of terminal differentiation marker proteins in vivo was also corroborated by in vitro studies with primary cultures of keratinocytes from the three different genotypes of mice. Twice weekly promotion treatments with TPA induced terminal differentiation of $\mathrm{CatE}^{+/+}$keratinocytes accompanying the expression of CatE and terminal differentiation marker genes in a manner associated with differentiation. The maximal expression of CatE was observed at $72 \mathrm{~h}$ after TPA treatment, suggesting its predominant expression in differentiating and/or differentiated keratinocytes. The expression profiles of K1, Loricrin and K13 were similar to that of CatE. Together, these results strongly suggest that CatE is positively involved in keratinocyte differentiation. The over-expression of CatE led to a significantly shortening of the time to reach the maximal expression of $K 1$ and $K 13$. In contrast, CatE deficiency resulted in a marked reduction in the expression of $K 1$, loricrin and K13, accompanying the impaired differentiation of keratinocytes. The present data thus indicate that CatE functionally links to the expression of such TPA-mediated keratinocyte terminal differentiation markers as K1, loricrin and K13 and thereby regulates the terminal regulation of keratinocytes. 


\section{Materials and Methods}

\section{Chemicals and Reagents}

DMBA and TPA were purchased from Sigma-Ardrich, Inc. (St. Louis, MO). Rabbit polyclonal antibodies against K1 (ab24643), K13 (ab58744) and loricrin (ab24772) was purchased from Abcam Inc. (Cambridge, MA). CnT-07 was from CELLnTEC Advanced Cell Systems (Bern, Switzerland). TrypLE Express was from GIBCO (Gaithersberg, MD). Annexin V-FLUOS Staining Kit and High Pure RNA Isolation Kit were from Roch Diagnostics GmbH (Basel, Switzerland). High Capacity cDNA Reverse Transcription Kit was from Applied Biosystems (Foster City, CA).

\section{Mice and Cells}

All animals were maintained according to the guidelines of the Japanese Pharmacological Society in a specific pathogen-free facility at the Kyushu University Station for Collaborative Research. All animal experiments were approved by the Animal Research Committee of the Graduate School of Dental Science, Kyushu University. Wild-type and $\mathrm{Cat}^{-/-}$mice on the C57BL/6 genetic background were used as described previously (Tsukuba et al., 2003). Transgenic mice overexpressing CatE $\left(C a t E^{T g}\right)$ on the C57BL/6 genetic background were generated according to the method 
as described (Kawakubo et al., 2007). Three different genotypes of the syngeneic mice were fertile and exhibited normal bleeding behavior and no obvious phenotypes when raised under specific pathogen-free conditions.

\section{Skin Treatments}

Each genotype of mice at 8-9 weeks of age was assigned to two treatment groups of eight mice each. Only those mice were in the resting phase of hair cycle were used. Lower dorsal skin of each genotype was shaved with a hair-clipper, and then DMBA (400 nmol) in acetone was applied on an approximately 2-cm area. The control group was received acetone alone. After 1 week, twice-weekly topical application of $20 \mathrm{nmol}$ TPA in $0.1 \mathrm{ml}$ of acetone was begun and continued for 14 weeks. Tumors were defined as raised lesions of at least $1 \mathrm{~mm}$ diameter that had been present for at least one week. The first tumors that appear during a two-stage mouse skin tumorigenesis protocol using DMBA and TPA as the initiator and promoter, respectively, are premalignant lesions called papillomas (reviewed in Klein-Szanto, 1989). The papillomas that initially develop during mouse skin initiation-promotion protocols have long been considered heterogeneous in that some will persist, some will disappear or regress, and only a small portion will progress to an invasive squamous cell carcinomas (reviewed in DiGiovanni, 1992). Indeed, the conversion of papillomas to carcinomas was too 
infrequent in any genotype to allow meaningful comparisons under the conditions used.

Papillomas formed were thus mapped and counted weekly.

\section{In situ hybridization (ISH) for CatE gene}

Skin and stomach of each genotype of C57Bl/6 mice at 12 weeks of age were dissected, fixed with Tissue Fixative (Genostaff Co., Ltd., Tokyo, Japan), embedded in paraffin, and then sectioned at $4 \mu \mathrm{m}$. In situ hybridization was performed on these sections using digoxigenin-labeled RNA probes (Roche Applied Science) under contract by Genostaff. Probes for a 360-base pair fragment were designed from position 176 to 535 of the open reading frame of mouse CatE. Hybridization was carried out as described previously (Fujiwara et al., 2004). For probe preparation, a mouse cDNA for CatE was amplified by RT-PCR using the gene-specific primer set. digoxigenin-labeled antisense and sense RNA probe was prepared by in vitro transcription with T7 RNA polymerase (DIG RNA Labeling Kit, Roche), using amplified cDNA of the gene as a template. Targeted mRNA sequence position for mouse CatE (NM_00779) was 176-535 (360 nt). The probes were hybridized at $60{ }^{\circ} \mathrm{C}$ with $60 \%$ formamide. Sections were treated with anti-digoxigenin antibody at room temperature and visualized with diaminobenzidine.

\section{Immunohistochemistory}

Surgical specimens were fixed in $10 \%$ neutral formalin for $24 \mathrm{~h}$ at room temperature, 
embedded in paraffin at $55^{\circ} \mathrm{C}$ and cut into parallel consecutive 3- $\mu \mathrm{m}$-thick sections for the subsequent immunohistochemical study using Simple Stain Mouse MAX-PO (Nichirei Biosciences Inc., Tokyo, Japan). Briefly, following deparaffinized in xylene, the sections were pretreated with autoclave for antigen retrieval in TE buffer (10 mM Tris-HCl, $1 \mathrm{mM}$ EDTA (pH 9.0)). After washed with PBS, endogenous peroxidase activity was then blocked with $3 \% \mathrm{H}_{2} \mathrm{O}_{2}$ in methanol for $10 \mathrm{~min}$, and following several washes, blocking reagent was applied for 60 min to prevent unspecific reactions. Sections were successively incubated with primary antibody against K1, K13, and Loricrin (1: 100 dillution) at $4{ }^{\circ} \mathrm{C}$ overnight. Especially for K13, CSA II (Dako, Glostrup, Denmark), Biotin-Free Catalyzed Amplification System, was performed before DAB color development. Following another washes, the bound primary antibodies were visualized by peroxidase detection using MAX-PO(R) reagent and DAB reagent. All sections were counterstained with hematoxylin.

\section{Primary cultures of keratinocytes}

Primary cultures of keratinocytes were generated from each genotype of newborn mice. Neonatal skin was taken from each genotype and incubated in CnT-07 supplement with $5 \mathrm{mg} / \mathrm{ml}$ dispase at $4{ }^{\circ} \mathrm{C}$ overnight. Following several washes with $\mathrm{CnT}-07$, epidermis was gently separated from dermis, and then placed on a $500 \mu 1$ drop of TrypLE Express 
(GIBCO, Gaithersberg, MD) in petri dishes at room temperature for $25 \mathrm{~min}$. After several washes with CnT-07, keratinocytes were collected and centrifuged, and then seeded in dishes at $4.0 \times 10^{4}$ cells $/ \mathrm{cm}^{2}$, and cultured under humidified $5 \% \mathrm{CO}_{2}$ environment. Afterwards, unattached cells were removed by washing with PBS and attached cells were further cultured in fresh medium, which was replaced every 3 day. For analysis of cell proliferation, primary cultures of keratinocytes from each genotype were seeded at $3 \times 10^{5}$ cells in $35 \mathrm{~mm} \mu$-dish (ibidi GmbH, Martinsried, Germany) and cultured to reach at $80 \%$ confluence, and then incubated in the presence or absence of DMBA (200 and $400 \mathrm{nM}$ ) at $37{ }^{\circ} \mathrm{C}$ for $24 \mathrm{~h}$ under humidified 5\% CO2 environment. After complete removal of the culture medium, the attached cells were washed with PBS. The extent of viability of the remaining cells was assessed by an apoptosis/necrosis assay with an Annexin-V-FLUOS Staining Kit (Roche Diagnostics GmbH, Mannheim, Germany). The cells treated with the kit in HEPES buffer containing propidium iodide (PI) in the dark for $10 \mathrm{~min}$ at room temperature were analyzed by fluorescence microscopy. For analysis of cell differentiation, primary cultures of keratinocytes from each genotype were incubated with TPA (40 nM) for various time intervals at $37{ }^{\circ} \mathrm{C}$ under humidified 5\% CO2 environment. The extent of differentiation was assessed by the expression profiles of various differentiation marker 
proteins by quantitative RT-PCR.

\section{Real-time RT-PCR}

Total RNA was extracted by High Pure RNA Isolation Kit (Roche), and two-step real-time qPCR was carried out using Taqman real-time PCR system on Light Cycler 480 instrument (Roche). cDNA was prepared from 500 ng total RNA in $20 \mu$ l reaction volume using High Capacity cDNA Reverse Transcription Kit (Applied Biosystems, Foster City, CA). Amplicon sizes were confirmed using RT-PCR, concretely GAPDH (75 bp), CatE (61 bp), K1 (127 bp), K13 (99 bp), and Loricrin (76 bp). Primers to amplify the following transcripts are as follows: GAPDH: tgtccgtcgtggatctgac (forward), cctgcttcaccaccttcttg (reverse), cathepsin E: aacctctgggtcccttctgt (forward), ggaatactgggtgtgcttg (reverse), Keratin1: tgagctgaagaacatgcaaga (forward), catgtaagctgaatccacatcc (reverse), Keratin13: ggagctccggatcaagatt (forward), ttgagcctgaagtcatctgc (reverse), and Loricrin: ggttgtggaaagacctctgg (forward), agccgccaccgctataat (reverse). Light Cycler Universal Probe Master (Roche) specific for each sequence were applied No.80 (for GAPDH and K13), No.26 (for CatE), No.31 (for K1), and No.63 (for Loricrin), respectively.

\section{Immunoblot analysis}

SDS-PAGE and immunoblot analysis were performed as described previously (Tsukuba 
et al., 2006).

\section{Statistical analysis}

The statistical significance was determined by unpaired Student $t$-test. A p-value of $<0.05$ was considered statistically significant.

\section{Acknowledgements}

This study was supported in part by a Grant-in-Aid for Scientific Research (B) from the Ministry of Education, Culture, Sports, Science, and Technology of Japan. 


\section{References}

Aldaz, C.M., Conti, C.J., Klein-Szanto, A.J.P., Slaga, T.J. (1987) Progressive dysplasia and aneuploidy are hallmarks of maouse skin pappilomas: relevance to malignancy. Proc. Natl. Acad. Sci. U.S.A. 84, 2029-2032.

Athauda, S.B.P., Takahashi, T., Inoue, H., Ichinose, M., Takahashi, K. (1991) Proteolytic activity and cleavage specificity of cathepsin $\mathrm{E}$ at the physiological $\mathrm{pH}$ as examined towards the B chain of oxidized insulin. FEBS Lett. 292, 53-56.

Blanpain, C., Fuchs, E. (2009) Epidermal homeostasis: a balancing act of stem cells in the skin. Nat. Rev. Mol. Cell Biol. 10, 207-217.

Burns, F.J., Vanderlaan, M., Sivak, A., Albert, R.E. (1976) Regression kinetics of mouseskin papillomas. Cancer Res. 36, 1422-1427.

Candi, E., Schmidt, R., Melino, G. (2005) The cornified envelope: a model of cell death in the skin. Nat. Rev. Mol. Cell Biol.6, 328-340.

Chavanas, S., Bodemer, C., Rochat, A., Hamel-Teillac, D., Ali, M., et al. (2000) Mutations of SPINKS5, encoding a serine protease inhibitor, cause Netherton syndrome. Nat. Genet. 25, 141-142.

Demerjian, M., Hachem, J-P., Tschachler, E., Denecker, G., Declercq, W., Vandenabeele, P., Mauro, T., Hupe, M., Crumrine, D., Roelandt, T., Houben, E., Elias, P.M., 
Feingold, K.R. (2008) Acute modulations in permeability barrier function regulate epidermal cornification. Am. J. Pathol. 172, 86-97.

Dhawan, D., Balasubramanian, S., Amonkar, A.J., Singh, N. (1999) Chemopreventive effect of 4-methyl epipodophyllotoxin on DMBA/TPA-induced mouse skin carcinogenesis. Carcinogenesis 20, 997-1003.

DiGiovanni, J. (1992) Multistage carsinogenesis in mouse skin. Pharmac. Ther. 54, 63-128.

Dlugosz, A.A., Yuspa, S.H. (1993) Coordinate changes in gene-expression which mark the spinous to granular-cell transition in epidermis are regulated by protein kinase-C. J. Cell Biol. 120, 217-225.

Egberts, F., Heinrich, M., Jensen, J-M., Winoto-Morbach, S., Pfeiffer, S., Wickel, M., Schunck, M., Steude, J., saftig, P., Proksch, E., Schütze, F. (2004) Cathepsin D is involved in the regulation of transglutaminase 1 and epidermal differentiation. $\mathrm{J}$. Cell Sci. 117, 2295-2307.

Fu, W., McCormick, T., Qi, X., Luo, L., Zhou, L., Li, X., Wang, B-C., Gibbons, H.E.,

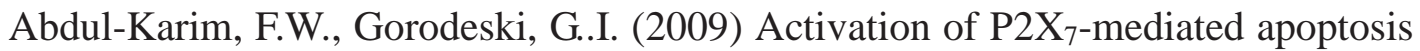
inhibits DMBA/TPA-induced formation of skin papillomas and cancer in mice. BMC Cancer 9, 114-133. 
Fujiwara, K., Ochiai, M., Ohta, T., Ohki, M., Aburatani, H., Nagao, M., Sugimura, T., Nakagama, H. (2004) Global gene expression analysis of rat colon cancers induced by a food-borne carcinogen, 2-amino-1-methyl-6-phenylimidazo[4,5-b]pyridine.

Carcinogenesis. 25, 1495-1505.

Gimenez-Conti, I., Aldaz, CM., Bianchi, AB., Roop, DR., Slaga, TJ., Conti, CJ. (1990)

Early expression of type I K13 keratin in the progression of mouse skin papillomas. Carcinogenesis. 11, 1995-1999.

Guo, L., Degrenstein, L., Fuchs, E. (1996) Keratinocyte growth factor is required for hair development but not for wound healing. Gene Dev. 10, 165-175.

Hara, K., Fukuyama, K., Sakai, H., Yamamoto, K., Epstein, W.L. (1993) Purification and immunohistochemical localization of aspartic proteinases in rat epidermis. J. Invest. Dermatol. 100, 394-399.

Henningsson, F., Yamamoto, K., Saftig, P., Reinheckel, T., Peters, C., Knight, S.D., Pejler, G. (2005) A role for cathepsin E in the processing of mast-cell carboxypeptidase A. J. Cell Sci. 118, 2035-2042.

Hébert, J.M., Rosenquist, T., Götz, J., Martin, G.R. (1994) FGF5 as a regulator of the hair growth cycle: Evidence from targeted and spontaneous mutations. Cell 78, 1017-1025. 
Huber, M., Rettler, I., Bernasconi, K., Frenk, E., Lavvrijsen, S.P., Ponec, M., Bon, A., Lautenschlager, S., Schorderet, D.F., Hohl, D. (1995) Mutations of keratinocyte transglutaminase in lamellar ichithyosis. Science 267, 525-528.

Igarashi, S., Takizawa, T., Takizawa, T., Yasuda, Y., Uchiwa, H., Hayashi, S., Brysk, H., Robinson, J.M., Yamamoto, K., Brysk, M.M. (2004) Cathepsin D, but not cathepsin E, degrades desmosomes during epidermal desquamation. Br. J. Dermatol. 151, 355-361.

Ishimatsu-Tsuji, Y., Moro, I., Kishimoto, J. (2005) Expression profiling and cellular localization of genes associated with the hair cycle induced by wax depilation. J. Invest. Dermatol. 125, 410-420.

Kageyama, T. (1993) Rabbit procathepsin E and cathepsin E. Nucleotide sequence of cDNA, hydrolytic specificity for biologically active peptides and gene expression during development. Eur. J. Biochem. 216, 717-728.

Kageyama, T., Ichinose, M., Yonezawa, S. (1995) Processing of the precursors to neurotensin and other bioactive peptides by cathepsinE. J. Biol. Chem. 270, 19135-19140.

Kakehashi, H., Nishioku, T., Tsukuba, T., Kadowaki, T., Nakamura, S., Yamamoto, K. (2007) Differential regulation of the nature and functions of dendritic cells and 
macrophages by cathepsin E. J. Immunol 179, 5728-5737.

Kartasova, T., Roop, DR., Holbrook, KA., and Yuspa, SH. (1993) Mouse differentiation-specific keratin 1 and 10 require a preexisting keratin scaffold to form a filament network. J. Cell Biol. 120, 1251-1261.

Kawakubo, T., Okamoto, K., Iwata, J., Shin, M., Okamoto, Y., Yasukochi, A., Nakayama, K.I., Kadowaki, T., Tsukuba, T., Yamamoto, K. (2007) Cathepsin E prevents tumor growth and metastasis by catalyzing the proteolytic release of soluble TRAIL from tumor cell surface. Cancer Res. 67, 10869-10878.

Kim, DJ., Kataoka, K., Sano, S., Connolly, K., Kiguchi, K., and DiGiovanni, J. (2009) Targeted disruption of Bcl-xL in mouse keratinocytes inhibits both UVB- and chemically induced skin carcinogenesis. Mol. Carcinog. 48, 873-885.

Klein-Szanto, A.J.P. (1989) Pathology of human and experimental skin tumors. In: Skin Tumors: Experimental and Clinical Aspects. Pp. 19-53, Conti, C.J., Slaga, T.J., and Klein-Szanto, A.J.P. (eds) Raven Press, New York.

Leder, A., Kuo, A., Cardiff, R.D., Sinn, E., Leder, P. (1990) V-Ha-Ras transgene abrogates the initiation step in mouse skin tumorigenesis - effects of phorbol esters and retinoic acid. Proc. Natl. Acad. Sci. USA 87, 9178-9182.

Lees, W.E., Kalinska, S., Meech, J., Capper, S.J., Cook, N.D., Kay, J. (1990) Generation 
of human endothelin by cathepsin E. FEBS Lett. 273, 99-1-2.

List, K., Haudenschild, C.C., Szabo, R., Chen, W., Wahl, S.M., Swaim, W., Engelholm, L.H., Behrendt, N., Bugge, T.H. (2002) Matriptase/MT-SP1 is required for postnatal survival, epidermal barrier function, hair follicle development, and thymic homeostasis. Oncogene 21, 3765-3779.

Muller-Rover, S.B., Handjiski, C., van der Veen, S., Eichmuller, K., Foitzik, I.A., McKay, K.S., Stenn, K.S., Paus, R. (2001) A comprehensive guide for the accurate classification of murine hair follicles in distinct hair cycle stages. J. Invest. Dermatol. 117, 3-15.

Ovaere, P., Lippens, S., Vandennabeele, P., Declercq, W. (2009) The emerging roles of serine protease cascades in the epidermis. Trends Biochem. Sci. 34, 453-463.

Robles, AI., Gimenez-Conti, IB., Roop, D., Slaga, TJ., Conti, CJ. (1993) Low frequency of codon 61 Ha-ras mutations and lack of keratin 13 expression in 7, 12-dimethylbenz[a]-anthracene-induced hamster skin tumors. Mol. Carcinog. 7, 94-98.

Roth, W., Deussing, J., Botchkarev, V.A., Pauly-Eerts, M., Saftig, P., Hafner, A., Schmidt, P., Schmahl, W., Scherer, J., Anton-Lamprecht, I., Von Figura, K., Paus, R., Peters, C. (2000) Cathepsin L deficiency as molecular defect of furless: 
hyperproliferation of keratinocytes and perturbation of hair follicle cycle. FASEB J. 14, 2075-2086.

Russel, L.J., DiGiovanna, J.J., Rogers, G.R., Steinert, P.M.., Hashem, N., Compton, J.G., Bale, S.J. (1995) Mutations in the gene for transglutaminase 1 in autosomal recessive lamellar ichthyosis. Nat. Genet. 9, 279-283.

Sakai, H., Saku, T., Kato, Y., Yamamoto, K. (1989) Quantitation and immunohistochemical localization of cathepsins $\mathrm{E}$ and $\mathrm{D}$ in rat tissues and blood cells. Biochim. Biophys. Acta 991, 367-375.

Shin, M., Kadowaki, T., Iwata, J., Kawakubo, T., Yamaguchi, N., Takii, R., Tsukuba, T., Yamamoto, K. (2007) Association of cathepsin E with tumor growth arrest through angiogenesis inhibition and enhanced immune responses. Biol. Chem. 388, $1173-1181$.

Stenn, K.S., Paus, R. (2001) Controls of hair follicle cycling. Physiol. Rev. 81, 449-494. Sundberg, J.P., Erickson, A.A., Roop, D.R., and Binder, R.L. (1994) Ornithine decarboxylase expression in cutaneous papillomas in SENCAR mice is associated with altered expression of Keratins 1 and 10. Cancer Res. 54, 1344-1351.

Sundberg, J.P., Boggess, D., Hogan, M.E., Sundberg, B.A., Rourk, M.H., Harris, B., Johnson, K., Dunstan, R.W., Davisson, M.T. (1997) Harlequin ichthyosis (ichq): a 
juvenile lethal mouse mutation with ichthyosiform dermatitis. Am. J. Pathol. 151, 293-310.

Sutter, C., Nischt, R., Winter, H., and Schweizer, J. (1991) Aberrant in vitro expression of keratin K13 induced by Ca2+ and vitamin A acid in mouse epidermal cell lines. Exp. Cell Res. 195, 183-193.

Tiano, HF., Loftin, CD., Akunda, J., Lee, CA., Spalding, J., Sessoms, A., Dunson, DB., Rogan, EG., Morham, SG., Smart, RC., and Langenbach, R. (2002) Deficiency of either Cyclooxygenase (COX)-1 or COX-2 alters epidermal differentiation and reduces mouse skin tumorigenesis. Cancer Res. 62, 3395-3401.

Toomes, C., James, J., Wood, A.J., Wu, C.L., McCormick, D., et al. (1999) Loss-of-function mutations in the cathepsin $\mathrm{C}$ gene results in periodontal disease and palmoplantar keratosis. Nat. Genet. 23, 421-424.

Tsukuba, T., Okamoto, K., Okamoto, Y., Yanagawa, M., Kohmura, K., Yasuda, Y., Uchi, H., Nakahara, T., Furue, M., Nakayama, K., Kadowaki, T., Yamamoto, K., Nakayama, K.I. (2003) Association of cathepsin E deficiency with development of atopic dermatitis. J. Biochem. 134, 893-902.

Tsukuba, T., Yamamoto, S., Yanagawa, M., Okamoto, K., Okamoto, Y., Nakayama, K.I., Kadowaki, T., Yamamoto, K. (2006) Cathepsin E-deficient mice show increased 
susceptibility to bacterial infection associated with the decreased expression of multiple cell surface Toll-like receptors. J. Biochem. 140, 57-66.

Tsukuba, T., Ikeda, S., Okamoto, K., Yasuda, Y., Sakai, E., Kadowaki, T., Sakai, H., and Yamamoto, K. (2006) Characterization of rat cathepsin E and mutants with changed active-site residues and lacking propeptides and N-glycosylation, expressed in human embryonic kidney 293T cells. FEBS J. 273, 219-229.

Yamamoto, K. (1999) Cathepsin E and cathepsin D. In: Proteases: New Perspectives, V. Turk, ed. (Basel, Switzerland; Birkhäuser Verlag), pp. 59-71.

Yanagawa, M., Tsukuba, T., Nishioku, T., Okamoto, Y., Okamoto, K., Takii, R., Terada, Y., Nakayama, K.I., Kadowaki, T., Yamamoto, K. (2007) Cathepsin E deficiency induces a novel form of lysosomal storage disorder showing the accumulation of lysosomal membrane sialoglycoproteins and the elevation of lysosomal $\mathrm{pH}$ in macrophages. J. Biol. Chem. 282, 1851-1862.

Zeeuwen, P.L., Vlijmen-Willems, I.M., Hendriks, W., Merkx, G.F., Schalkwijk, J. (2002) A null mutation in the cystatin $\mathrm{M} / \mathrm{E}$ gene of ichq mice causes juvenile lethality and defects in epidermal cornification. Hum. Mol. Genet. 11, 2867-2875.

Zeeuwen, P.L.I.M. (2004) Epidermal differentiation:the role of proteases and their inhibitors. Eur. J. Cell Biol. 83, 761-773. 


\section{Figure legends}

Figure 1. Impairment of differentiation in the epidermis and hair follicle in $\mathrm{CatE}^{-/}$mice and CatE expression in these tissues in wild-type littermates. (A) Histological analysis of surgical skin specimens from $\mathrm{CatE}^{-/-}$and $\mathrm{CatE}^{+/+}$mice at 3 weeks of age. The paraffin sections were processed for hematoxylin and eosin staining. The skin of $\mathrm{CatE}^{-/-}$ mice was characterized by the significantly expanded corium and the significantly reduced subcutaneous tissue and the hair follicles in the anagen stage. Bars, $100 \mu \mathrm{m}$. (B) Western blotting analysis of the cell extract of skin keratinocytes, as well as the serum, from $\mathrm{CatE}^{+/+}$mice with antibodies against rat CatE (1/500). Immunocomplexes on the nitrocellulose membranes were detected with chemiluminescence reagents. (C) ISH analysis of mouse gastric mucosa with DIG-labeled antisense and sense RNA probes for mouse CatE. ISH with mouse gastric mucosa was performed for validation of the probes used. Prominent expression of Cat E in stomach, especially in epithelial cells was observed, as would be expected. (D) ISH analysis of hair follicles of $\mathrm{CatE}^{+/+}$ mice.CatE was expressed around inner root sheath of hair follicles.

Figure 2. Histopathology of skin from each genotype of mice initiated with DMBA and promoted with twice weekly treatment of TPA for 14 weeks. The photographs from top to bottom in each column represent the representative histological sections stained with hematoxylin and eosin of papillomas in $\mathrm{CatE}^{-/-}, \mathrm{CatE}^{+/+}$, and $\mathrm{CatE}^{T g}$ mice, respectively. 
The photographs from left to right in each column show low and higher magnification of the respective histological sections of papillomas in each genotype. The specimens in each row are the same magnification. Histological analyses revealed that all tumors from each genotype were squamous cell papillomas, but not squamous cell carcinomas. Black and white arrowheads indicate mitosis and tumor-infiltrated neutrophils, respectively. Data are representative of resultss obtained with eight animals for each group.

Figure 3. Papillomas occurring in the three different genotypes of mice treated with DMBA and TPA. Each genotype of skin was initiated with 400 nmol DMBA and promoted with twice weekly treatments of 20 nmol TPA. (A) Gross appearance of lesions in each genotype. Data are representative of photographs obtained with eight animals for each group. (B) Papilloma incidence per mouse was plotted against time. The data are means from eight animals for each group. $\circ, \mathrm{CatE}^{-/} ; \bullet, \mathrm{CatE}^{+/+} ; \mathbf{\Delta}$, $\operatorname{CatE}^{T g}$ (C) Number of papillomas per mouse was plotted against time. ○, CatE $^{-/-}$; •, $\mathrm{CatE}^{+/+} ; \boldsymbol{\Lambda}, \mathrm{CatE}^{\mathrm{Tg}}$ (D) Distribution of the size of papillomas generated in each genotype at 14 weeks after TPA promotion was plotted as white $\left(\right.$ CatE $\left.^{-/}\right)$. Black $\left(\mathrm{CatE}^{+/+}\right)$and grey $\left(\mathrm{CatE}^{\mathrm{Tg}}\right)$ columns. The data are means from eight animals for each group. 
Figure 4. Immunohistochemical detection of $\mathrm{K} 1$ (A), loricrin (B) and $\mathrm{K} 13$ (C) in sections of papillomas generated in each genotype of mice initiated with PMA and promoted with twice weekly treatment of TPA for 14 weeks. Sections were stained by the avidin-biotin complex method with primary antibodies directed to each marker protein and by nuclei-staining hematoxylin. Data are representative of results obtained with eight animals for each group. Control indicates the sections from the wild-type mice treated with acetone without DMBA and TPA. The photographs in each right column represent higher magnification views of the images (squares) in the corresponding left column. Arrows and white arrowheads indicate epidermis and muscle layer, respectively.

Figure 5. Effect of DMBA on primary cultures of keratinocytes prepared from the three different genotype of syngeneic mice. Cultured keratinocytes from each genotype was incubated with acetone alone and DMBA (200 and $400 \mathrm{nM}$ ) for $24 \mathrm{~h}$ and then subjected to an apoptosis/necrosis assay with Annexin-V-FLUOS (green) and PI staining kits. Scale bars, $50 \mu \mathrm{m}$.

Figure 6. Western blotting analysis of primary cultures of keratinocytes from $\mathrm{CatE}^{+/+}$ mice treated with TPA. The cell extract of keratinocytes at the indicated times after TPA treatment were subjected to SDS-PAGE under reducing conditions and followed by 
1

2

3

4

5

7

8

10

11

12

13

14

15

16

17

18

19

20

21

22

23

24

25

26

27

28

29

30

31

32

33

34

35

36

37

38

39

40

41

42

43

44

45

46

47

48

49

50

51

52

53

54

55

56

57

58

59

60

61

62

63

64

65

immunoblotting with antibodies against CatE or actin. 


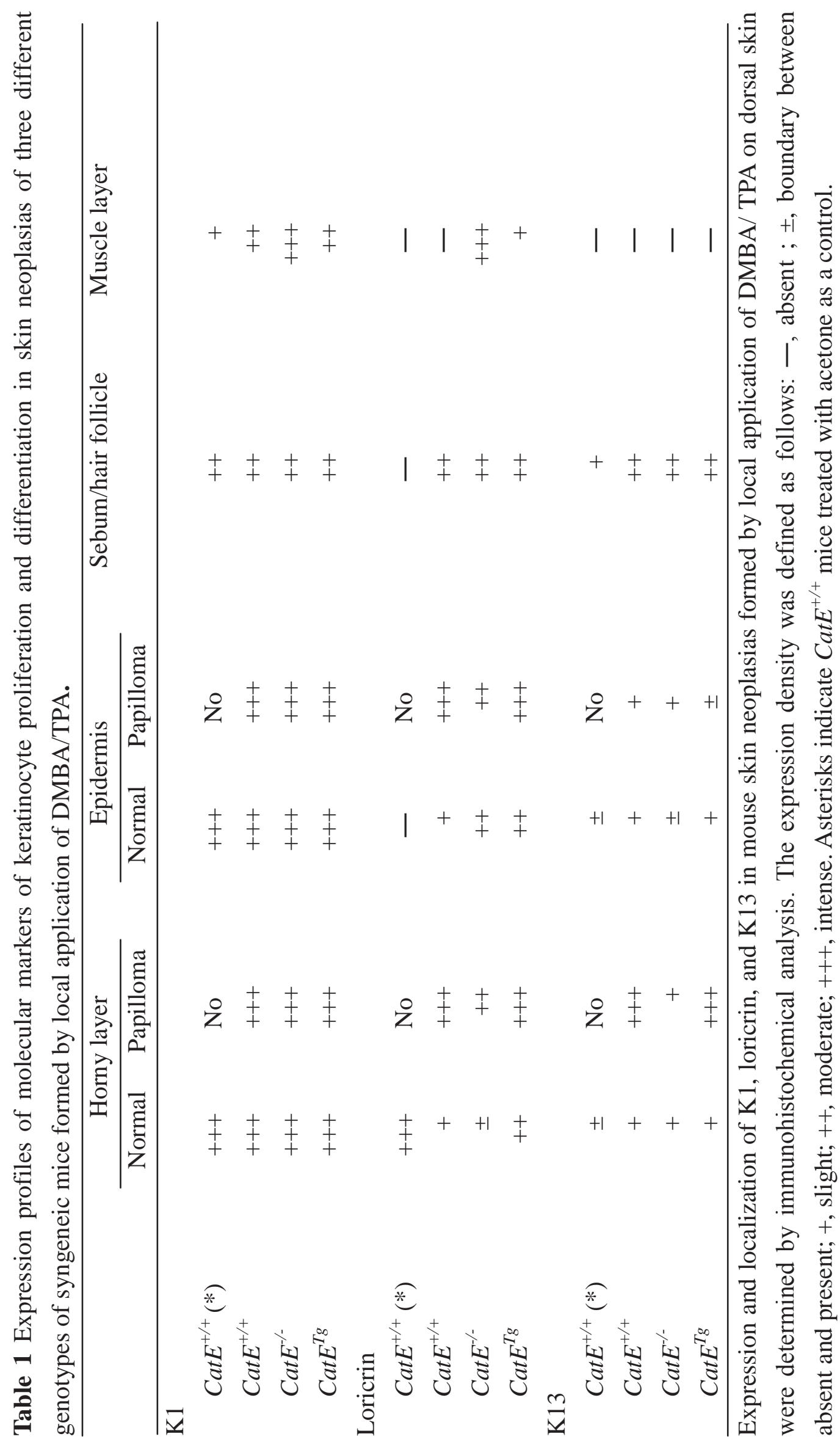




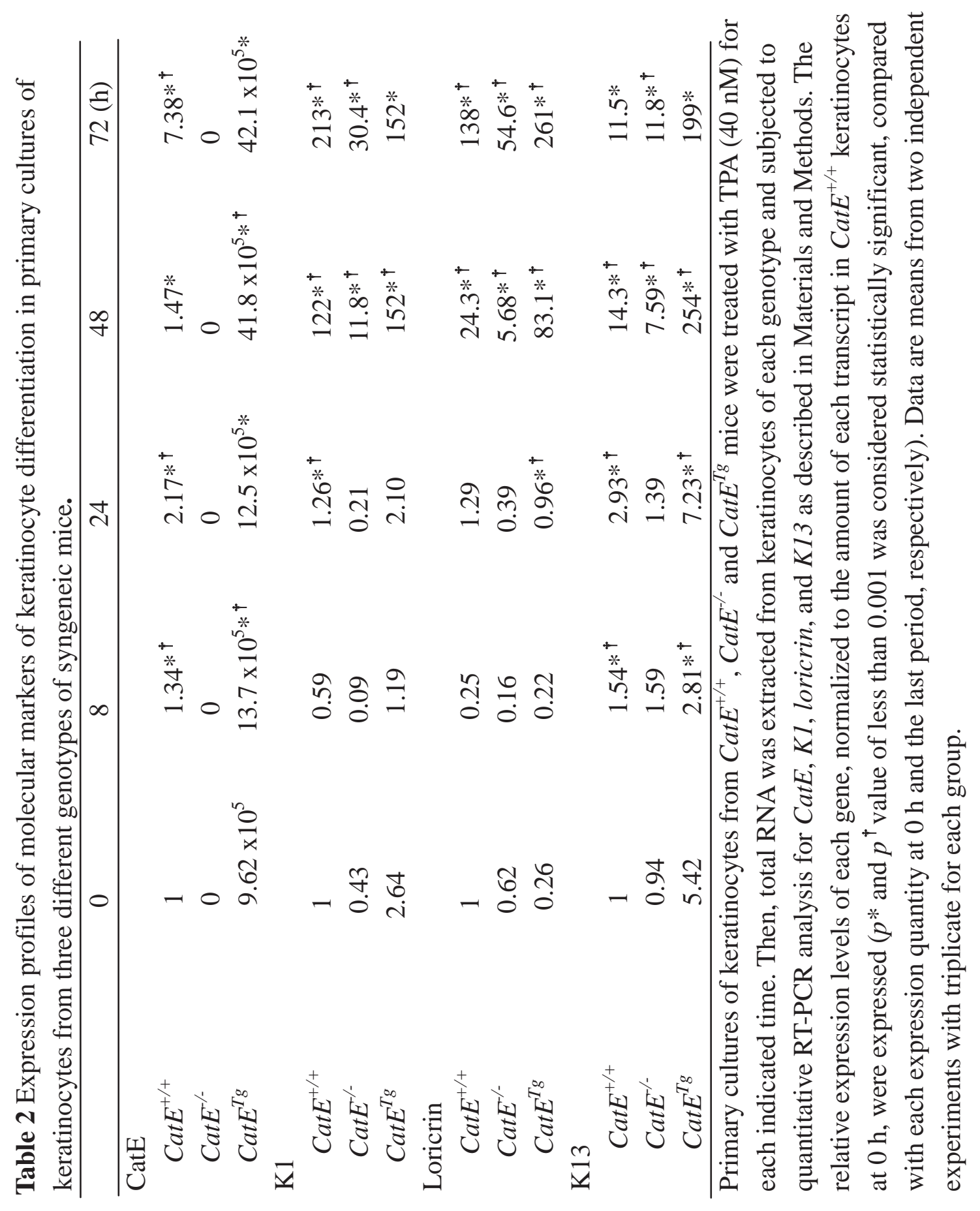


Figure 1
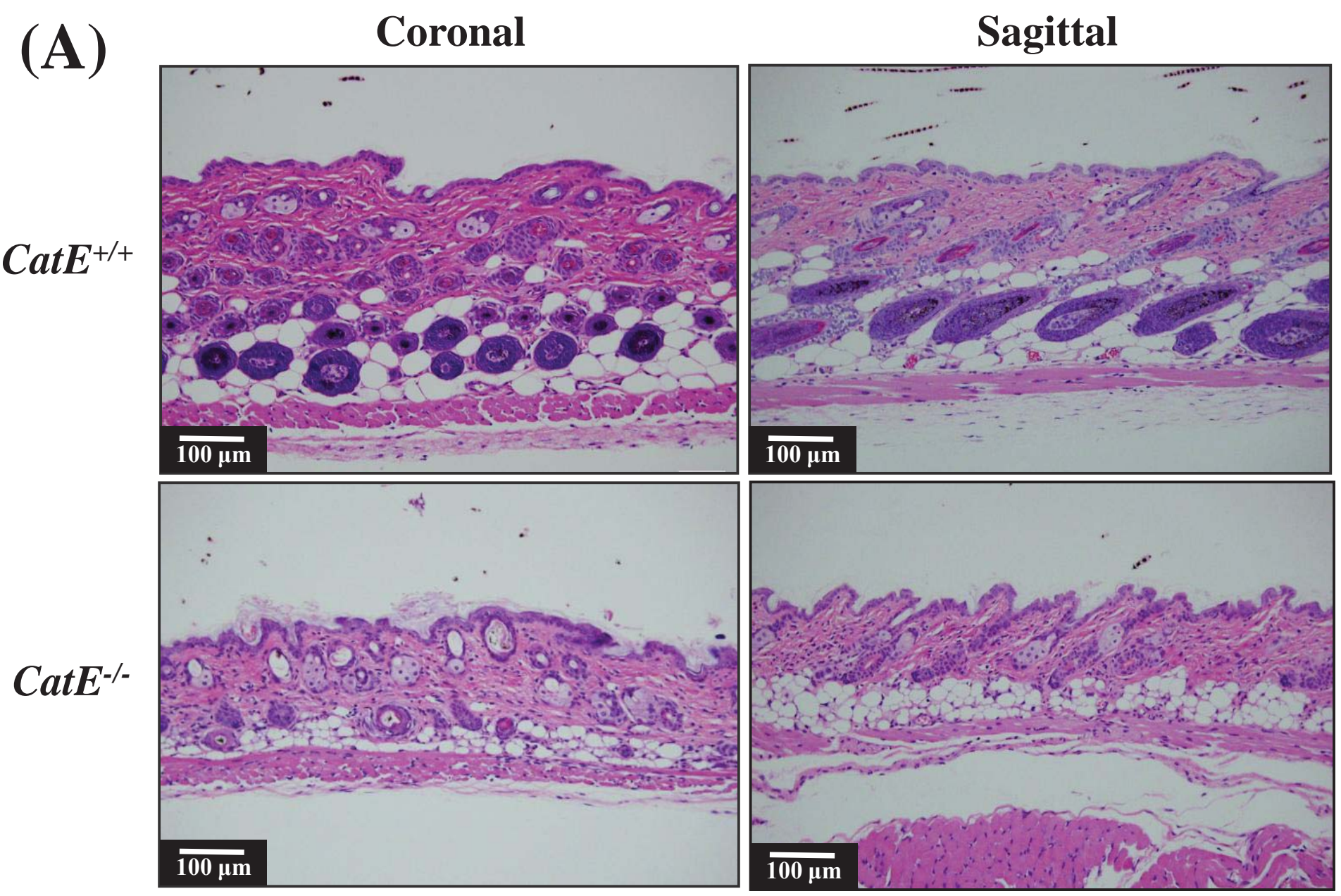

Fig. 1 Kawakubo et al. 

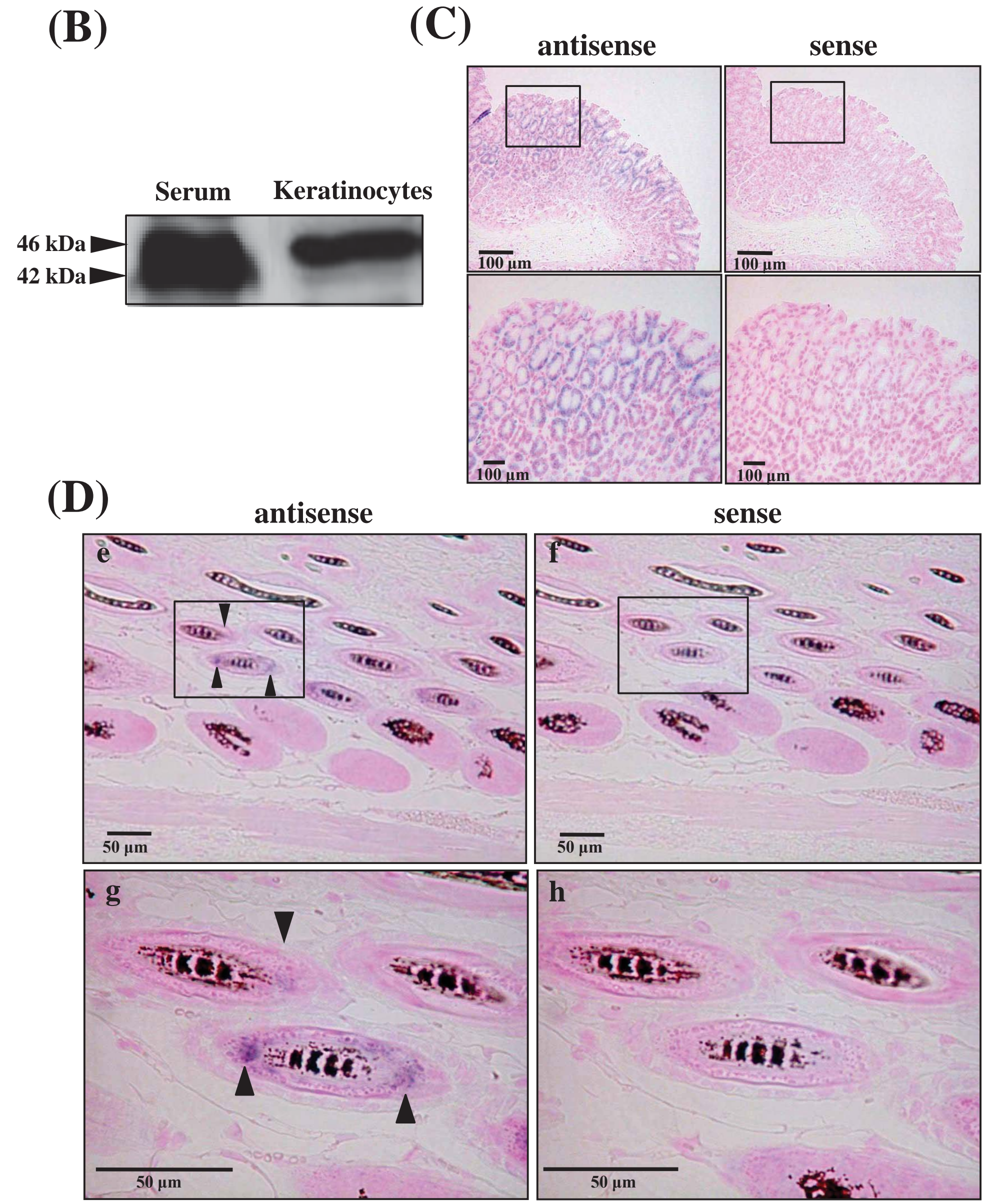

Fig. 1 Kawakubo et al. (continued) 


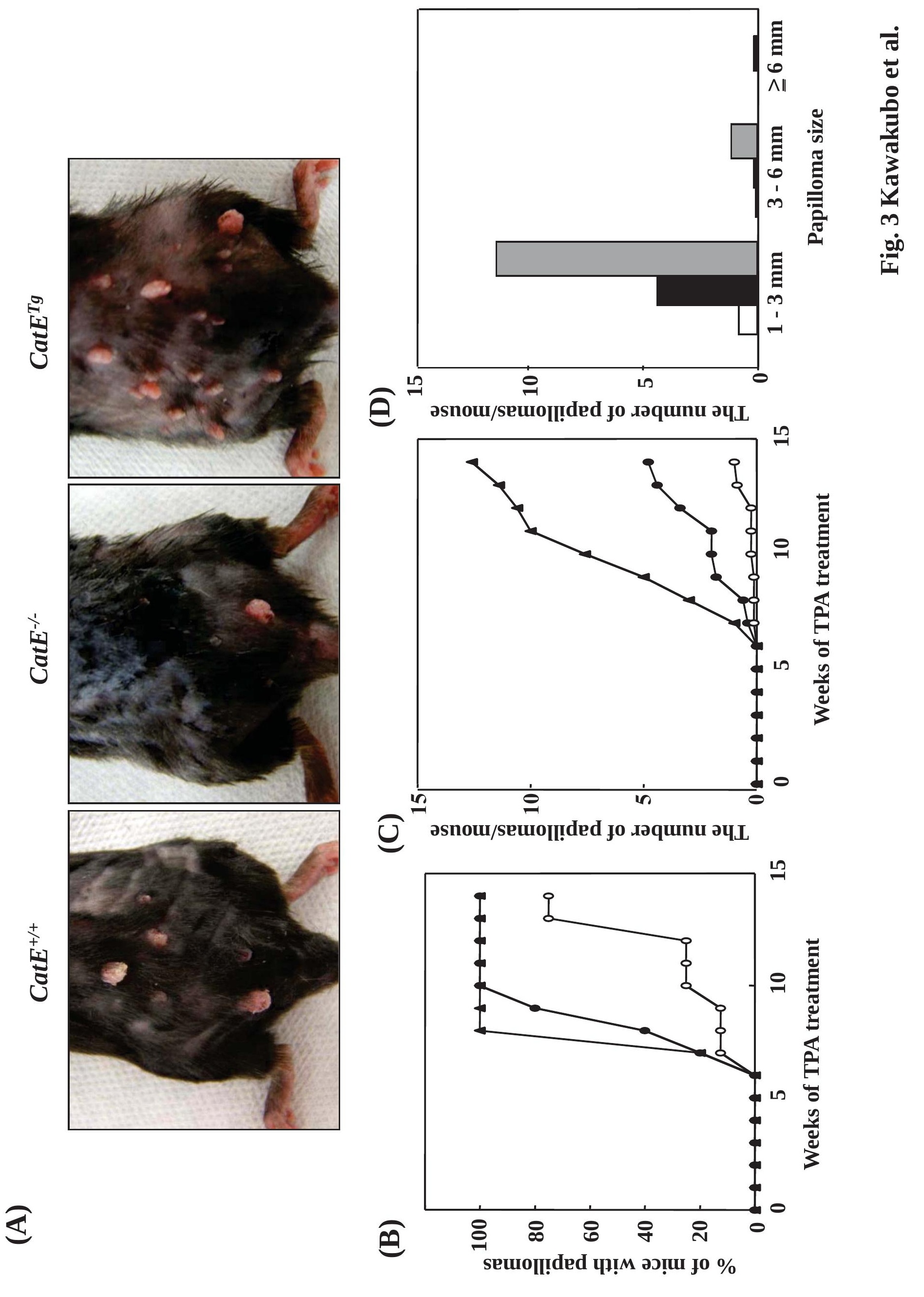



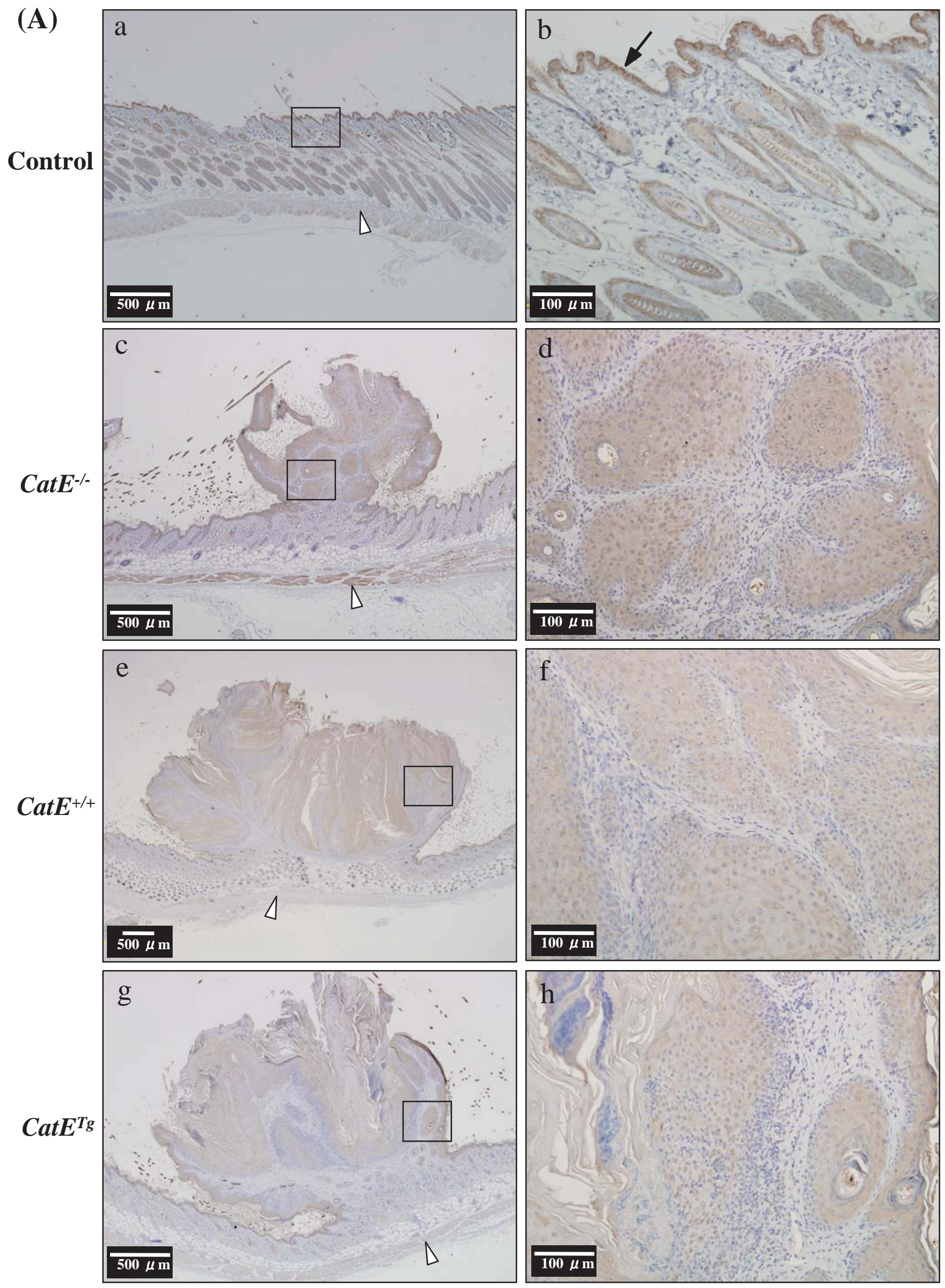

Fig. 4 Kawakubo et al. 

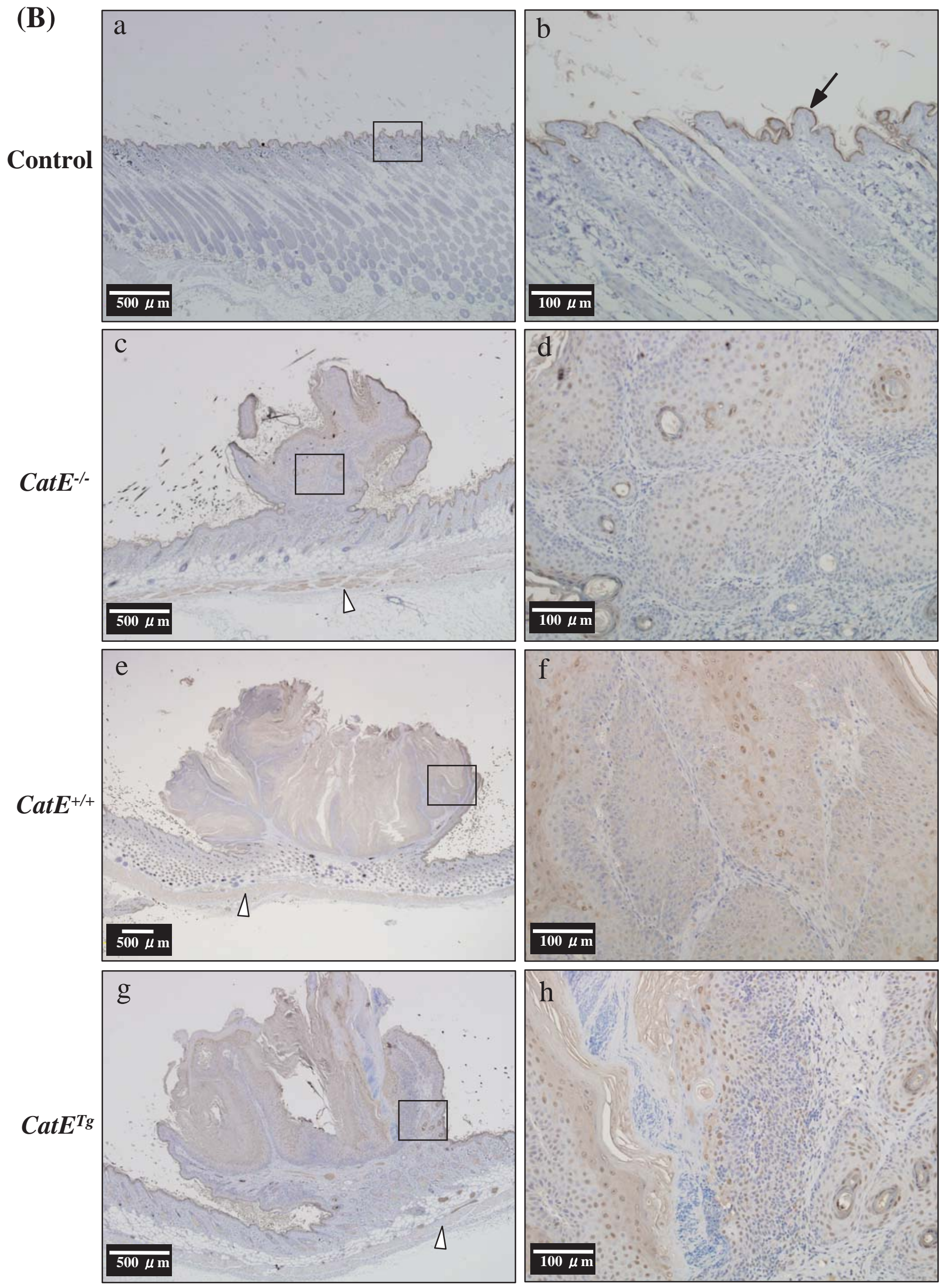

Fig. 4 Kawakubo et al. (Continued) 

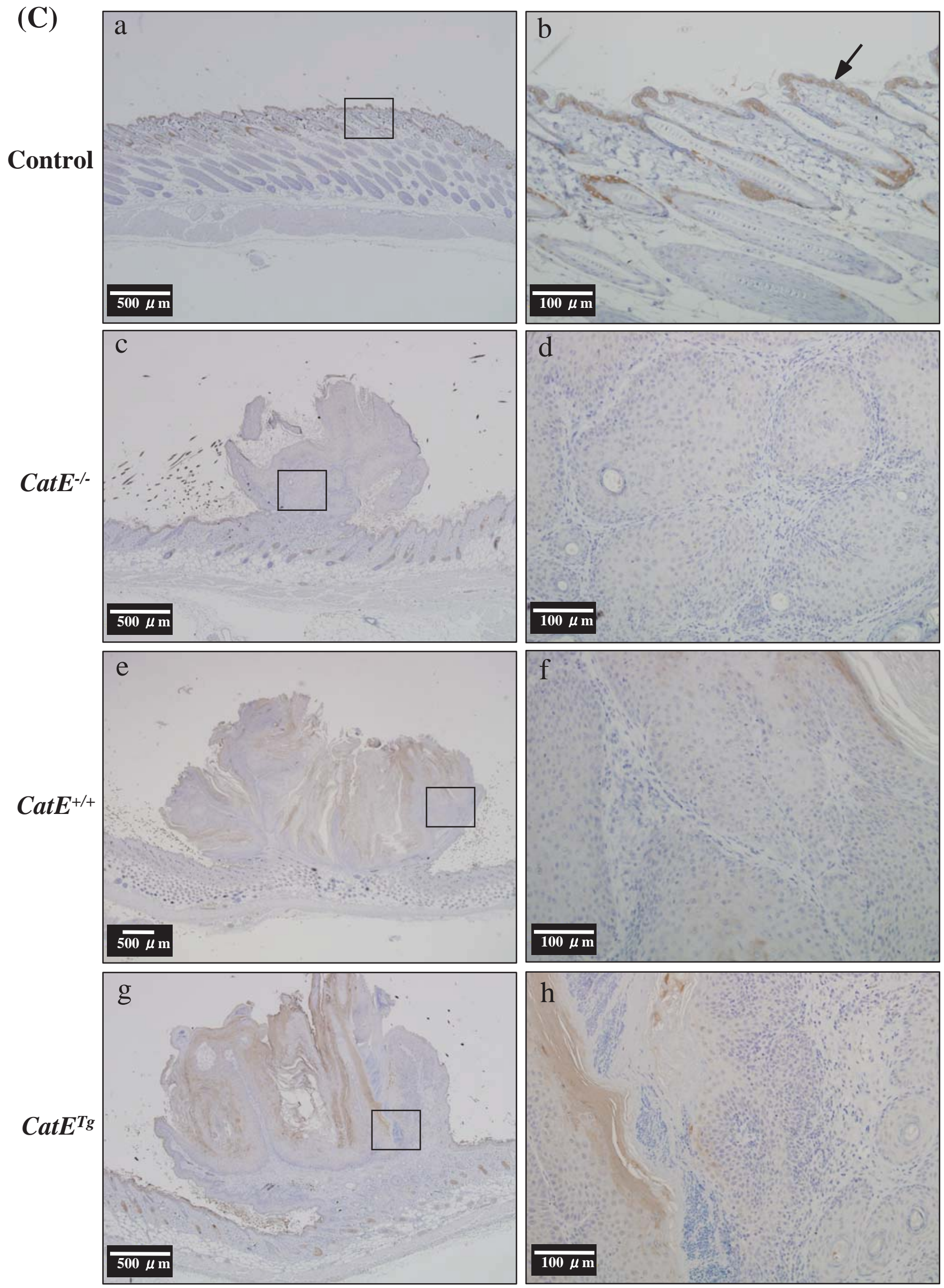

Fig. 4 Kawakubo et al. (Continued) 

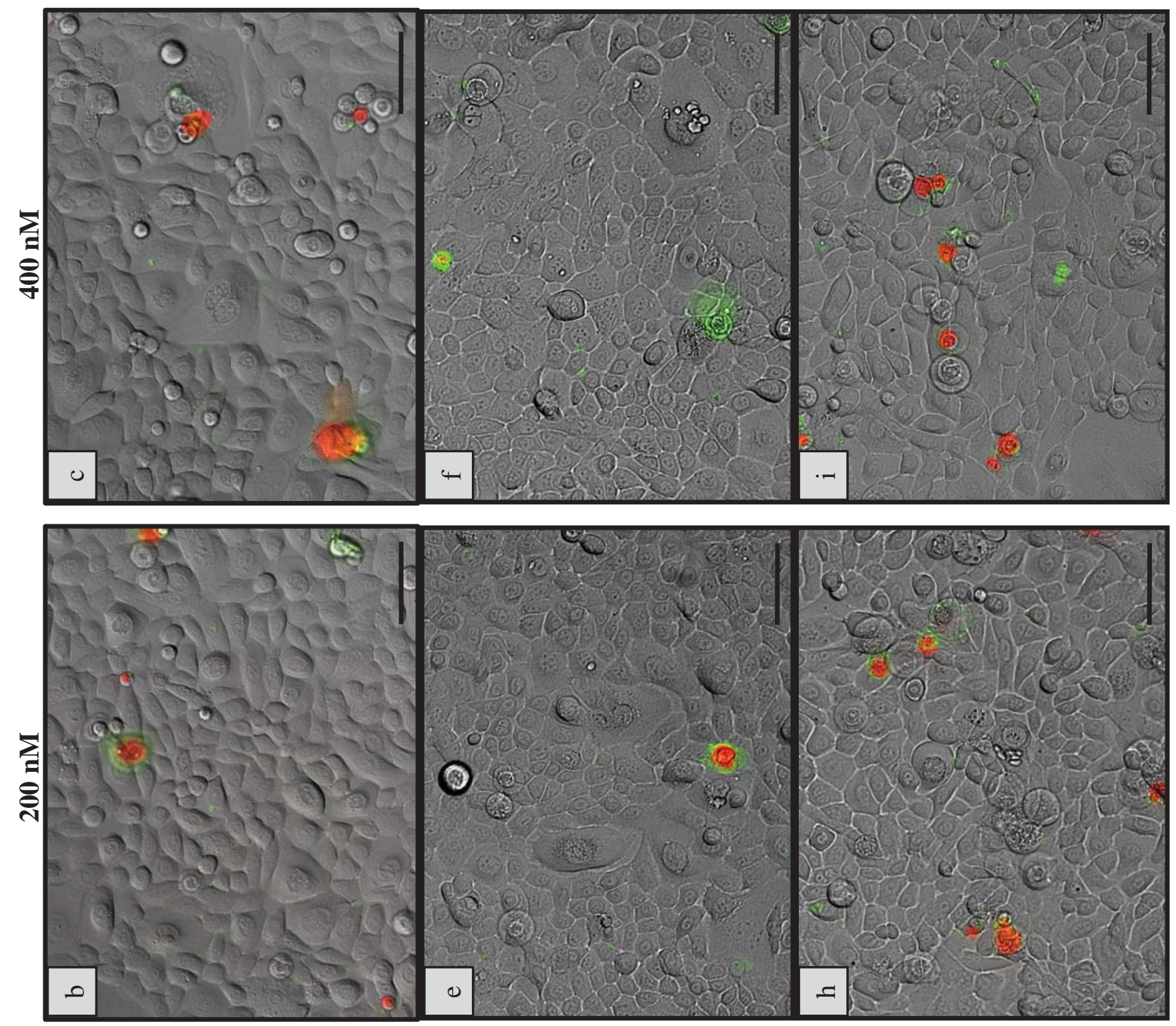

ปี่

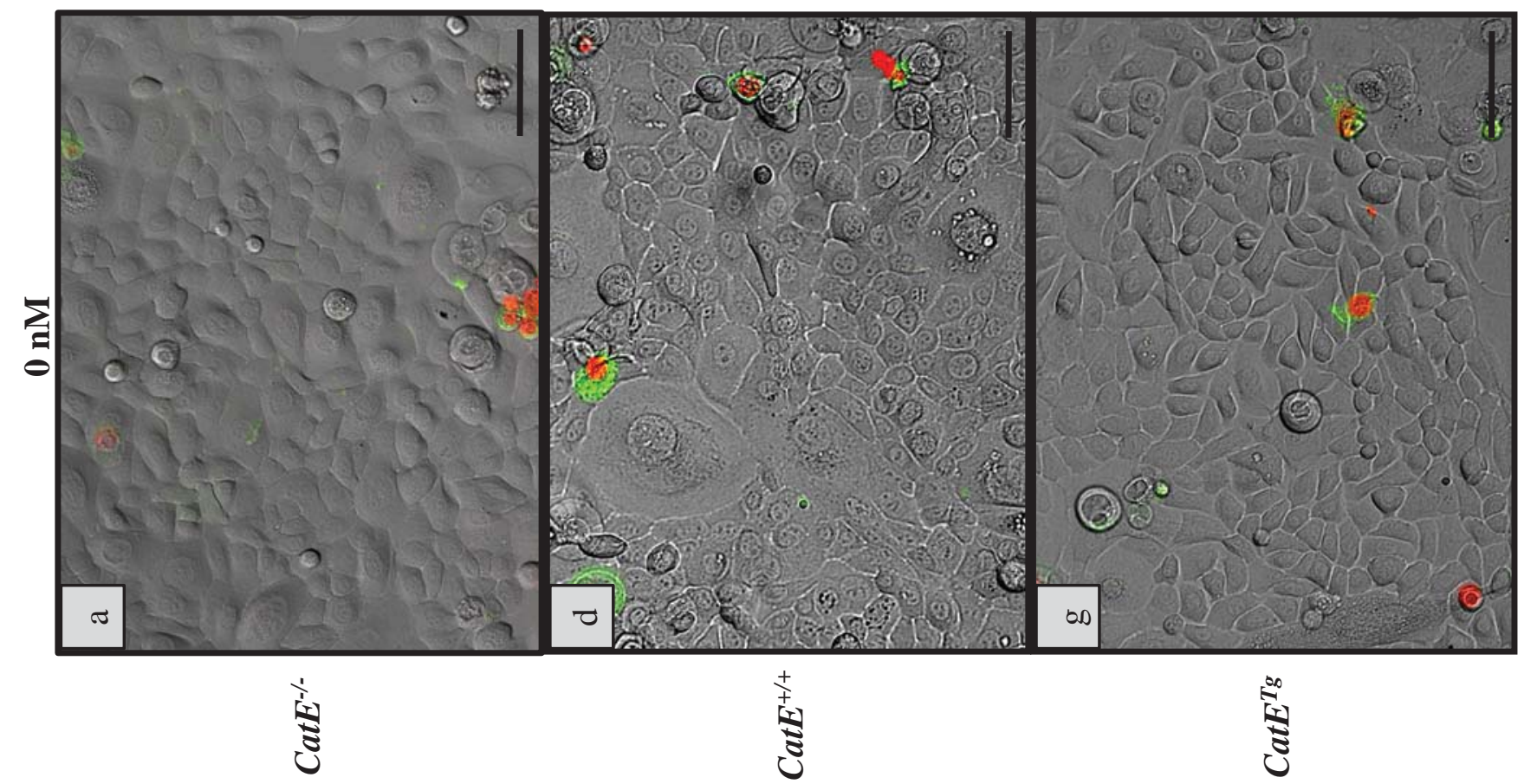

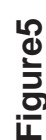




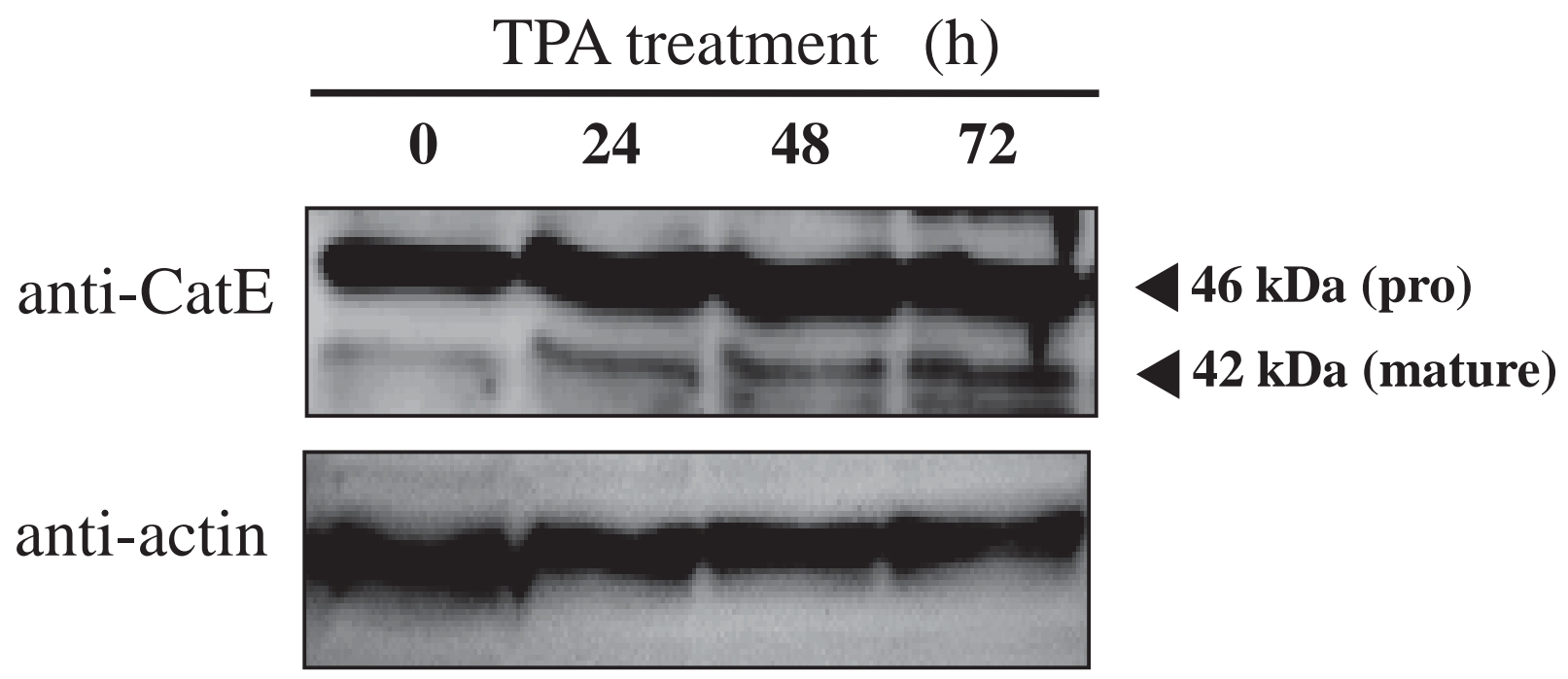

Fig. 6 Kawakubo et al. 